\title{
Coupling ratio tuning of direct UV-written X-couplers for cascaded power splitters in WDM networks
}

\author{
F.R. Mahamd Adikan, J.C. Gates, C.B.E. Gawith and P.G.R. Smith \\ E mail:mfra@orc.soton.ac.uk
}

We present a study of low polarisation sensitivity 2 degrees X-couplers with $20 \%$ coupling ratio tuning via refractive index asymmetry, attaining 50:50 power splitting at $1550 \mathrm{~nm}$. The device has less than $5 \%$ coupling ratio variation over $30 \mathrm{~nm}$ in the $\mathrm{C}$-band and $<0.7 \mathrm{~dB}$ polarisation dependent loss.

Introduction: X-couplers are integrated optical devices formed by two optical waveguides crossing at an angle. They find applications in switching arrays, passive crossovers, and power couplers and offer a significant reduction in device footprint compared to conventional directional couplers. Direct UV writing (DUW) provides an attractive technique for fabricating $\mathrm{X}$-couplers that cross at very small angle by eliminating photolithography and etching. DUW works by translating a photosensitive sample beneath a focused UV laser to create a localised change in refractive index as described in [1]. Our system is based on a frequency doubled argon-ion laser emitting at $244 \mathrm{~nm}$ for use with 3-layer silica-on-silicon substrates with a germanium doped core layer. Having demonstrated small angle $\left(0.8^{\circ}-4^{\circ}\right) \mathrm{X}$-couplers with relatively low polarisation and wavelength sensitivity [2], this paper investigates the effect of asymmetry between the two UV written X-coupler arms in tuning coupling performance. 
Similar work has previously been investigated by Svalgaard for producing 50:50 y-branches in $1 \times \mathrm{N}$ splitters [3], mode evolution 3-dB couplers [4], and beam combiners [5].

Our goal is to employ asymmetry in tuning the coupling behaviour of $\mathrm{X}$ couplers, enabling fabrication of cascaded devices for use in WDM networks. DUW allows us two degrees of freedom in producing asymmetry; dimensional (waveguide width) asymmetry is achieved by defocusing the UV laser to change the writing spot size, and refractive index asymmetry is achieved by varying the writing speed. We show that coupling tunability of $\sim 20 \%$ in the Cband $\left(1530-1560 \mathrm{~nm}\right.$ ) is achieved by varying fluence by only $\sim 8 \mathrm{kJcm}^{-2}$ (a UV writing speed variation of just $1.5 \mathrm{~mm} /$ minute), resulting in $50: 50$ power splitting. We also investigate the resulting changes in polarisation dependent loss and polarisation sensitivity.

UV Writing the X-couplers: The X-couplers fabricated for this experiment each have a $2^{\circ}$ crossing angle $(\beta=29$, for which the second $\mathrm{s}$-bend was written with various fluence levels, creating asymmetry in terms of UV induced index increase (refer Fig. 1). Fluence is an expression for the energy exposed to the sample during the UV writing process as a function of laser power, spot size, and writing speed. The fluence of the first s-bend and all input and output ports was fixed at $\sim 25.5 \mathrm{kJcm}^{-2}$ and those of the second s-bend were varied from $17.0-25.5 \mathrm{kJcm}^{-2}$, the latter of which forms a symmetric structure for comparison. 
An ASE input signal passed via a polariser and polarisation controller was used to characterise the X-couplers. The input and output signals were fibre butt-coupled into/from each device via flat cleaved SMF-28 fibres tipped with index matching oil. Output power measurements were taken from both output ports (ports 3 and 4) for light launched into port 1 . This was then repeated for launch into port 2. The sample end faces were polished to optical quality prior to UV writing and repeat measurements were taken to average out variances in fibre coupling, and end face quality. The output power measured were used to calculate coupling ratio, given by the ratio of the coupled power to the total output power of the X-coupler (i.e. sum of coupled and though power).

Results and discussion: Fig. 2 shows the coupling ratio, insertion loss relative to the fibre-to-fibre loss of a straight channel waveguide, and polarisation dependent loss (PDL) performance of the X-couplers with polarised input ASE launched into Port 1.

Here, it is evident that the coupling ratio varies from a maximum of $\sim 70 \%$ (symmetric case) to $50 \%$ (highest asymmetry case). $50 \%$ coupling ratio is ideal for cascaded $1 \times \mathrm{N}$ splitting applications and the $\sim 20 \%$ coupling ratio tunability achieved highlights the viability of tunable X-couplers over C-band wavelengths. This stems from the coupling behaviour of X-couplers being dependent on the refractive index of the waist, where the two waveguides overlap. 
As the asymmetry level increases, so does the difference in coupling ratio for the two polarisation cases, with a maximum difference of $\sim 5 \%$. Polarisation dependent loss (PDL) also increases with asymmetry, up to $\sim 0.65 \mathrm{~dB}$. An interesting behaviour worth pointing out is the reduction in insertion loss of the device with asymmetry for TE polarisation. We believe this is due to the increase in mode conversion efficiency moving away from symmetry, ensuring adiabatic mode evolution across the coupler [4]. Very low PDL $(<0.2 \mathrm{~dB})$ and coupling ratio difference $(<1 \%)$ were displayed as we move closer to symmetry, highlighting the polarisation insensitivity of direct UV written X-couplers. This low polarisation dependence is possible due to the inexistence of under-etched regions in the vicinity where the two s-bends meet, as described in [2].

In Fig. 3, the wavelength dependency of the coupling ratio in the Cband region shows a less than $\pm 5 \%$ coupling ratio variation over $30 \mathrm{~nm}$ with $50 \%$ coupling at $1550 \mathrm{~nm}$ for the case with the highest asymmetry, illustrating good device stability across the wavelength region. The graph clearly shows the coupling ratio tuning, with the required $50 \%$ coupling ratio obtained with $8 \mathrm{kJcm}^{-2}$ fluence variation.

Conclusions: We present the performance of asymmetric direct UV written X-couplers, describing the minor trade-offs between coupling tunability and polarisation dependency. Tailoring of waveguide asymmetry has been shown to tune coupling ratio by $20 \%$ with stable operation over $30 \mathrm{~nm}$ C-band spectral range. As such, the ability to manipulate the waist index by forming 
an etched window over the waist and manipulating the index via liquid crystal for example, would be a way to beam switching.

\section{References:}

[1] M. Svalgaard and M. Kristensen, "Directly UV written silica-on-silicon planar waveguides with low loss," Electronics Letters, vol. 33, pp. 861, 1997.

[2] F. R. M. Adikan, C. B. E. Gawith, P. G. R. Smith, I. J. G. Sparrow, G. D. Emmerson, C. Riziotis, and H. Ahmad, "Design and demonstration of direct UV-written small angle X-couplers in silica-on-silicon for broadband operation," Applied Optics, 2006.(accepted for publication)

[3] M. Olivero and M. Svalgaard, "UV-written integrated optical 1xN splitters," Optics Express, vol. 14, pp. 162-170, 2006.

[4] W. K. Burns, A. F. Milton, A. B. Lee, and E. J. West, "Optical modal evolution 3-dB coupler," Applied Optics, vol. 15, pp. 1053, 1976.

[5] D. A. Smith, J. E. Baran, J. L. Jackel, R. E. Wagner, and R. Welter, "A mode-evolution-type integrated-optical beam combiner for coherent receivers," IEEE Photonics Technology Letters, vol. 3, pp. 339-341, 1991.

\section{Authors' affiliations:}

F.R. Mahamd Adikan, J.C. Gates, C.B.E. Gawith and P.G.R. Smith (Optoelectronics Research Centre (ORC), University of Southampton, SO17 1BJ Southampton, United Kingdom) mfra@orc.soton.ac.uk 
Figure captions:

Fig. 1 Schematic representation of the fabricated UV written X-couplers with $2^{\circ}$ crossing angle. For asymmetric structures, the second s-bend arm is written with a different fluence value to that of the first s-bend arm.

Fig. 2 Coupling ratio and insertion loss of X-couplers with various second sbend UV writing fluence values highlighting the effects of index asymmetry of the structure (solid and dotted lines: curves of best fit).

- TE

\ $\mathrm{TM}$

- PDL

Fig. 3 Wavelength dependency of coupling ratio with various second s-bend fluence values (dotted lines: curves of best fit).

- $\quad$ Fluence, $F=25.5 \mathrm{kJcm}^{-2}$

- $F=21.8 \mathrm{kJcm}^{-2}$

- $F=19.1 \mathrm{kJcm}^{-2}$

- $\quad F=17.0 \mathrm{kJcm}^{-2}$ 


\section{Figure 1}

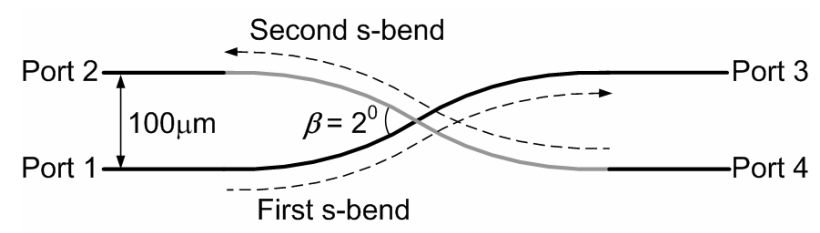

Output power

\begin{tabular}{c|c|c} 
Launch & Through & Coupled \\
\hline Port 1 & Port 3 & Port 4 \\
Port 2 & Port 4 & Port 3
\end{tabular}


Figure 2
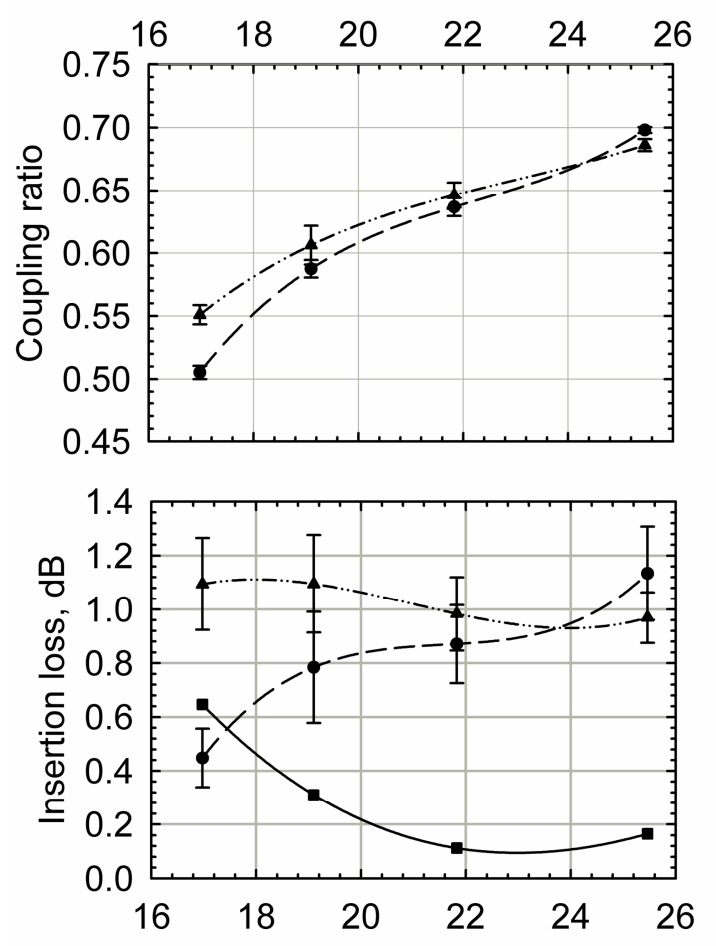

Fluence of second s-bend, $\mathrm{kJcm}^{-2}$ 
Figure 3

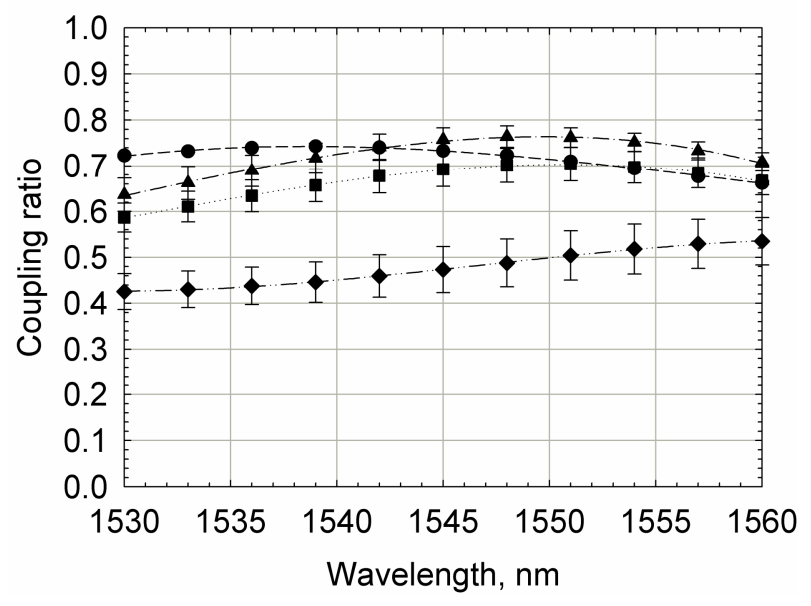

